\section{LB1.3 THE IMPACT OF HPV VACCINATION ON GENITAL WARTS IN ABORIGINAL AUSTRALIANS: ANALYSIS OF NATIONAL DATA}

${ }^{1} \mathrm{H}$ Ali ${ }^{*},{ }^{1,2,3} \mathrm{CC} \mathrm{O}^{\prime} \mathrm{Connor},{ }^{1} \mathrm{D}$ Callander, 'D Saulo, ${ }^{4} \mathrm{~S}$ Graham, ${ }^{1} \mathrm{M}$ Kong, ${ }^{1} \mathrm{DJ}$ Regan, ${ }^{1} \mathrm{AE}$ Grulich, ${ }^{5,6} \mathrm{CK}$ Fairley, ${ }^{1} \mathrm{RJ}$ Guy, ${ }^{1,7} \mathrm{~B}$ Donovan. ${ }^{1}$ The Kirby Institute, UNSW Australia, Sydney, NSW 2052, Australia; ${ }^{2}$ RPA Sexual Health, Sydney Local Health District, Sydney, NSW 2050, Australia; ${ }^{3}$ Central Clinical School, Sydney University, Sydney, NSW 2050, Australia; ${ }^{4}$ Centre for Epidemiology and Biostatistics, School of Population and Global Health, University of Melbourne, Parkville, VIC 3051, Australia; ${ }^{5}$ Melbourne Sexual Health Centre, Carlton, VIC 3053, Australia; ' 5 School of Population Health, University of Melbourne, Parkville, VIC 3010, Australia; ${ }^{7}$ Sydney Sexual Health Centre, Sydney Hospital, Sydney, NSW 2000, Australia

\subsection{6/sextrans-2015-052270.208}

Introduction Australia funded a national human papillomavirus (HPV) program for girls and young women (12-26 years) from 2007 and for young boys (12-15 years) from 2013. We evaluated the impact of the program in Aboriginal and Torres Strait Islander (Aboriginal) people who suffer disproportionately from HPV-related cancers.

Methods Routinely collected clinical data from 19 sexual health services in four jurisdictions were included. We calculated the proportion of Aboriginal attendees diagnosed with genital warts at first visit, before and after the start of the program, and compared this with non-Indigenous attendees. We calculated percentage change between time periods along with 95\% confidence intervals (CI). Final, clean and corrected datasets were received from the participating services in second quarter of 2015; after which the datasets were cleaned and data errors corrected in collaboration with the clinics before data could be analysed in July. Results From 2004-2014, 215,599 Australian born attendees were seen; of whom $7.2 \%$ identified as Aboriginal. The proportion of Aboriginal women aged $<21$ years diagnosed with warts decreased by $96.1 \%$ (95\% CI: 70.8\%-99.5\%) in the vaccination period, from $7.8 \%$ in 2007 to $0.3 \%$ in 2014 , comparable to the $90.8 \%$ (95\% CI: $85.5 \%-94.1 \%)$ decline in non-Indigenous women of the same age. The proportion of Aboriginal women aged 21-30 years diagnosed with warts decreased by $75.0 \%$ (95\% CI: $24.4 \%-91.9 \%$ ), from $6.0 \%$ to $1.5 \%$; comparable to the $75.4 \%$ (95\% CI: $68.9 \%-80.7 \%)$ decline in non-Indigenous women. The proportion of Aboriginal heterosexual men aged $<21$ years diagnosed with warts decreased from $7.3 \%$ in 2007 to no diagnosis in 2014 and among Aboriginal heterosexual men aged 21-30 years wart diagnoses decreased by $75.8 \%$ (95\% CI: $33.6 \%-91.3 \%)$, comparable to the decreases in $<21$ year old (88.7\%; 95\% CI: $79.5 \%-93.8 \%)$ and $21-30$ year old $(68.8 \%$; 95\% CI: $63.4 \%-73.5 \%)$ non-Indigenous men.

Conclusions Using genital warts as a proxy measure of the impact of the HPV vaccination program, Aboriginal and nonIndigenous Australians appear to have benefited equally.

\section{\begin{tabular}{|l|l}
\hline LB1.4 RTIES AMONG GAY MEN TAKING DAILY \\
\hline
\end{tabular} ANTIRETROVIRALS FOR PRE-EXPOSURE PROPHYLAXIS OF HIV: THE NSW DEMONSTRATION PROJECT PRELUDE}

${ }^{1}$ I Zablotska*, 'S Vaccher, ${ }^{1} \mathrm{C}$ Gianacas, ${ }^{1,2} \mathrm{G}$ Prestage, ${ }^{3} \mathrm{~A}$ McNulty, ${ }^{4} \mathrm{~J}$ Holden, ${ }^{4} \mathrm{H}$ Schmidt, ${ }^{1,5} \mathrm{DJ}$ Templeton, ${ }^{1} \mathrm{IM}$ Poynten, ${ }^{1} \mathrm{~A}$ Grulich, on behalf of the PRELUDE Study Team. ${ }^{1}$ Kirby Institute, University of New South Wales, Sydney, Australia; ${ }^{2}$ Australian Research Centre in Sex Health and Society, La Trobe University, Melbourne, Australia; ${ }^{3}$ Sydney Sexual Health Centre, Sydney, Australia; ${ }^{4}$ NSW Ministry of Health, Sydney, Australia; ${ }^{5}$ RPA Sexual Health, Sydney Local Health District, Sydney, Australia

10.1136/sextrans-2015-052270.209
Introduction Pre-exposure prophylaxis (PrEP) prevents HIV infections but not other STIs. We assessed prevalence and incidence of STIs among PrEP users in the NSW demonstration study, PRELUDE.

Methods By 14 July 2015, 268 gay and homosexually-identified men (GHM) were enrolled and started taking PrEP; 98 (36.6\%) and $27(10.1 \%)$ had reached three and six-month follow-up respectively. STI testing was conducted at baseline and quarterly follow-up visits. We calculated the baseline prevalence and incidence of STIs. The sample STI incidence will be compared with the population incidence in NSW.

Results At enrolment, 17.2\% of participants were diagnosed with $\geq 1$ STI, including $10.8 \%$ with rectal gonorrhoea, rectal chlamydia and/or syphilis. The latter three STIs were diagnosed in $2.6 \%, 7.8 \%$, and $1.1 \%$ of men, respectively. Among 98 men at 3-month follow-up, $17.3 \%$ were diagnosed with any of these STIs $3.1 \%$ had rectal gonorrhoea, $11.2 \%$ rectal chlamydia and $4.1 \%$ syphilis). At three months of follow-up, the incidence of these STIs was 18.4, 67.4 and 24.5 per 100 person-years (PY), respectively. By 14 July 2015, 27 PrEP users reached six months of follow-up. In this group, the incidence of rectal gonorrhoea was 29.6 per $100 \mathrm{PY}(11.2 \%$ increase from month three followup, $\mathrm{p}<0.04)$. No HIV infections were observed in the cohort. Conclusion Among PrEP users in PRELUDE, the baseline prevalence and incidence of STIs were high and remained high in the early follow-up (indeed, the incidence of gonorrhoea has increased). Despite enrolment in a clinical study, with frequent follow-up, STI testing and treatment, PrEP users remain at very high risk of STIs. Longer follow-up is necessary to assess whether STI trends among PrEP users will change. The investigation of prophylaxis against other STIs and other methods of testing and treating STIs among these high-risk men appears to be warranted.

Disclosure of interest statement The PRELUDE study is funded by the NSW Ministry of Health, with Gilead Sciences providing the study medication (Truvada).

\section{LB1.5 INITIAL INTERACTIONS OF HERPES SIMPLEX VIRUS WITH HUMAN SKIN DENDRITIC CELLS}

${ }^{1,2} \mathrm{M}$ Kim, ${ }^{1,2} \mathrm{NR}$ Truong, ${ }^{1} \mathrm{~V}$ James, ${ }^{1} \mathrm{~L}$ Bosnjak, ${ }^{1,2} \mathrm{KJ}$ Sandgren, ${ }^{1,2} \mathrm{AN}$ Harman, ${ }^{1,2} \mathrm{~N}$ Nasr, ${ }^{1,2} \mathrm{KM}$ Bertram, ${ }^{3} \mathrm{~N}$ Olbourne, ${ }^{4} \mathrm{~S}$ Sawleshwarkar, ${ }^{4} \mathrm{~K}$ McKinnon, ${ }^{5} \mathrm{RC}$ Cohen, ${ }^{1,2}$ AL Cunningham*. 'Westmead Millennium Institute for Medical Research; ${ }^{2}$ University of Sydney; ${ }^{3}$ Sydney Institute of Plastic and Reconstructive Surgery; ${ }^{4}$ Western Sydney Sexual Health Centre; ${ }^{5}$ TheChildren's Hospital at Westmead

\subsection{6/sextrans-2015-052270.210}

Introduction The mechanism by which immunity to herpes simplex virus (HSV) is initiated is not completely defined. HSV initially infects the stratified squamous epithelium of the anogenital mucosa prior to entering nerve endings. We have recently reported that topical application of HSV-1 to human foreskin explants results in infection of epidermal Langerhans cells (LCs) which then emigrate into the dermis where they expressed the maturation marker CD80 and formed large cell clusters with BDCA3 + subsets of DC-SIGN+ and dermal dendritic cells (DCs) and. HSV-expressing LC fragments were observed inside the dermal DCs/macrophages. No other infected epidermal cells interacted with the dermal DCs (Kim et al. Plos Pathogens, 2015).

Methods Therefore, we isolated LCs and dermal DCs from large abdominal skin specimens by collagenase digestion and flow 